\title{
Chromaticity Head-Tail application for the Tevatron Vahid Ranjbar
}

\begin{abstract}
The Chromaticity head-tail phase technique has been developed for use in the Tevatron. This method is based on the phase shift, which develops for particles of different energies within a single bunch. Since this phase shift is proportional to chromaticity, knowledge of the phase shift over a synchrotron period can allow extraction of the chromaticity. Currently the Tevatron uses a varying RF technique to measure chromaticity. So far the RF method cannot effectively measure chromaticity for coalesced proton bunches. The Head-Tail technique however seems to be capable of extracting chromaticity for coalesced protons even during acceleration. This application will greatly speed up chromaticity measurements especially during ramp and squeeze which currently require three separate ramps using uncoalesced protons.
\end{abstract}

\section{Introduction}

Under ideal circumstances a particle in an RF bucket will circulate in the bucket with the synchrotron frequency. Since chromaticity is a measure of the change in betatron tune vs. change in momentum, the betatron phase for a particle will depend on the chromaticity, the distance from the center of the bunch and the synchrotron frequency. Thus the betatron equation for a particle undergoing synchrotron oscillations in a bucket will become,

$\left.\mathrm{Z}(\mathrm{n})=\mathrm{A} \cos \left[2 \pi \mathrm{nQ}+\mathrm{Q}^{\prime} \omega \tau \cos \left(2 \pi \mathrm{n} \mathrm{Q}_{\mathrm{s}}\right)-1\right) / \eta\right]$

where $\mathrm{Z}(\mathrm{n})$ is the transverse position at turn $\mathrm{n}$ since the beam was kicked, $\mathrm{Q}$ is the betatron tune, $Q_{s}$ is the synchrotron tune, $\tau$ is the longitudinal position with respect to the center of the bunch, $Q$ ' is the chromaticity, $\omega$ the revolution frequency and $\eta$ the momentum compaction factor. If two longitudinal positions in the bunch are selected separated by $\Delta \tau$, the phase difference will become,

$\Delta \Psi(\mathrm{n})=-\mathrm{Q}^{\prime} \omega \Delta \tau \eta /\left[\cos \left(2 \pi \mathrm{n} \mathrm{Q}_{\mathrm{s}}\right)-1\right]$.

Thus we can solve for Q' to obtain, $Q^{\prime}=-\eta \Delta \Psi(n) / \omega \Delta \tau\left[\cos \left(2 \pi n Q_{s}\right)-1\right]$.

\section{Hardware and Software Set-up}

Using the 1-meter long strip line detector installed at F0 in the Tevatron the signal is captured using a Tektronics TDS7000 series oscilloscope. The A-B and A+B signals are measured with a resolution of $0.4 \mathrm{~ns}$ across $20 \mathrm{~ns}$ for 1049 turns. Since the recorded 
signal is the sum the image current traveling with the beam and the reflected image of the beam from the downstream end we first de-convolute the single image by subtracting out the reflected image. This is accomplished digitally using knowledge of the length of strip line and the velocity of the beam. . The set-up and an example of the de-convoluted sum signal can be seen in Fig. 1.

After reconstruction of the signal the transverse position is then determined by taking the ratio of the sum and difference signal,

$Z=27 x(A-B) /(A+B)$.

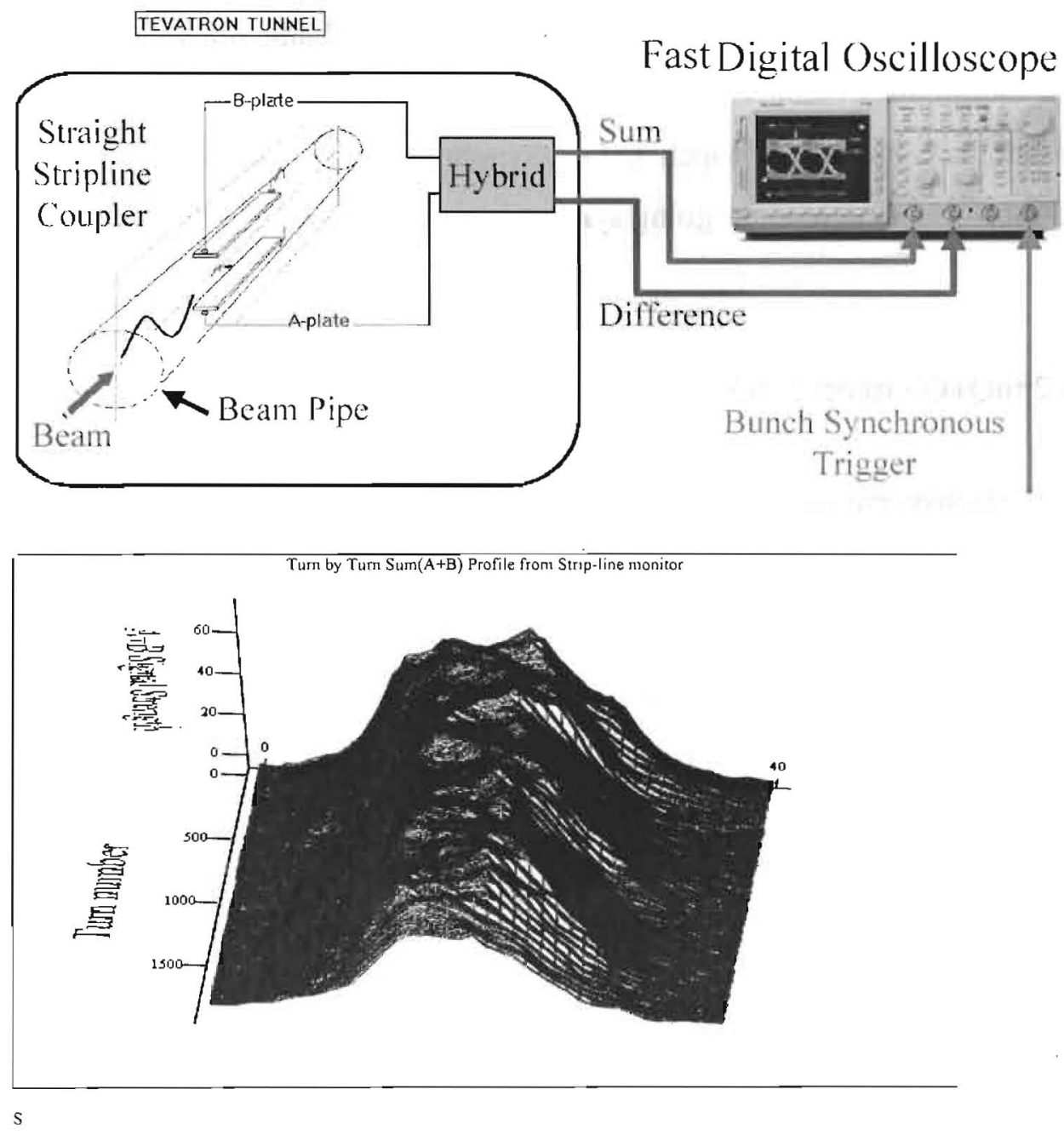

Figure 1: Using the vertical and horizontal strip-line detectors installed in the Tevatron at the F0 location we extract a profile of the transverse behavior of the beam over a single longitudinal bunch 
The $\mathrm{A}+\mathrm{B}$ signal is then used to identify the bunch center. We found that this was essential since for many data sets the bunch center would oscillate with the synchrotron frequency $Q_{s}$. An example of this bunch oscillation can be seen in Fig.2. We selected two longitudinal positions around the bunch center separated by $\Delta \tau=0.8$ nsecs from each other. According to extensive simulation work done at $\mathrm{CERN}^{1}$ this symmetrical location should mitigate any effects due to both non-linearities and acceleration.

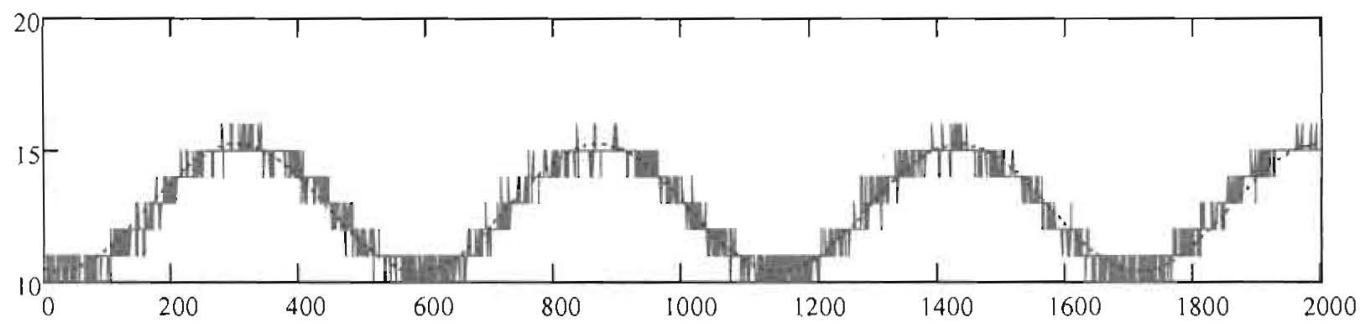

Figure 2: Bunch center oscillations after horizontal kick.

The scope has an onboard processor with a running a Lab View application. This Lab View program is in turn controlled by a vax console program (C100) via a few acnet variables: T:CHRMO (the acquisition state i.e. single or multiple measurements, coalesced or uncoalesced protons) and T:CHRMCN which set the scope to acquire data. $\mathrm{V}$ :CHROM returns the current state of a given measurement $(0-5), 2=$ scope is armed $5=$ measurement complete. The scope is fed a series of event driven triggers I:F1N8C6 and I:F1N8C5. T:TBSDD0 defines the trigger timing (.195 Trev yields the P0 bunch) and T:RTBSMT the number of triggers.

The Lab View front end both controls the scope and pre-processes the waveforms generating transverse position turn-by-turn data for the vertical and horizontal head and tail slices of a given bunch. These four position arrays are then read into acnet array variables: T:CHRXT, XH,YT,YH. 
The $\mathrm{C} 100$ vax console program controls the $\mathrm{E} 17$ vertical kicker and F17 horizontal injection kicker by setting the voltage of each kick and the timing as well as setting the events and delays. The E17 kicker uses a thyratron, which produced a half sinusoid current pulse, with a base width of $10 \mu$ secs. The $\mathrm{F} 17$ extraction kicker produces a square current pulse with a width of $1.8 \mu$ secs. Since the timing of the E17 vertical kicker varied with voltage, a timing versus voltage table was built into the software using observed kicker response time. This table is graphed in Fig. 3. Since we have a history of burning out the E17 thyratron tubes any future replacement tubes may require an adjustment of this voltage versus timing table. In addition the $\mathrm{C} 100$ program sets up the events and triggers for the scope (I:F1N8C6, I:F1N8C5, T:TBSDD0 and T:RTBSMT) and controls the state of the Lab View program via T:CHRMO and T:CHRMCN.

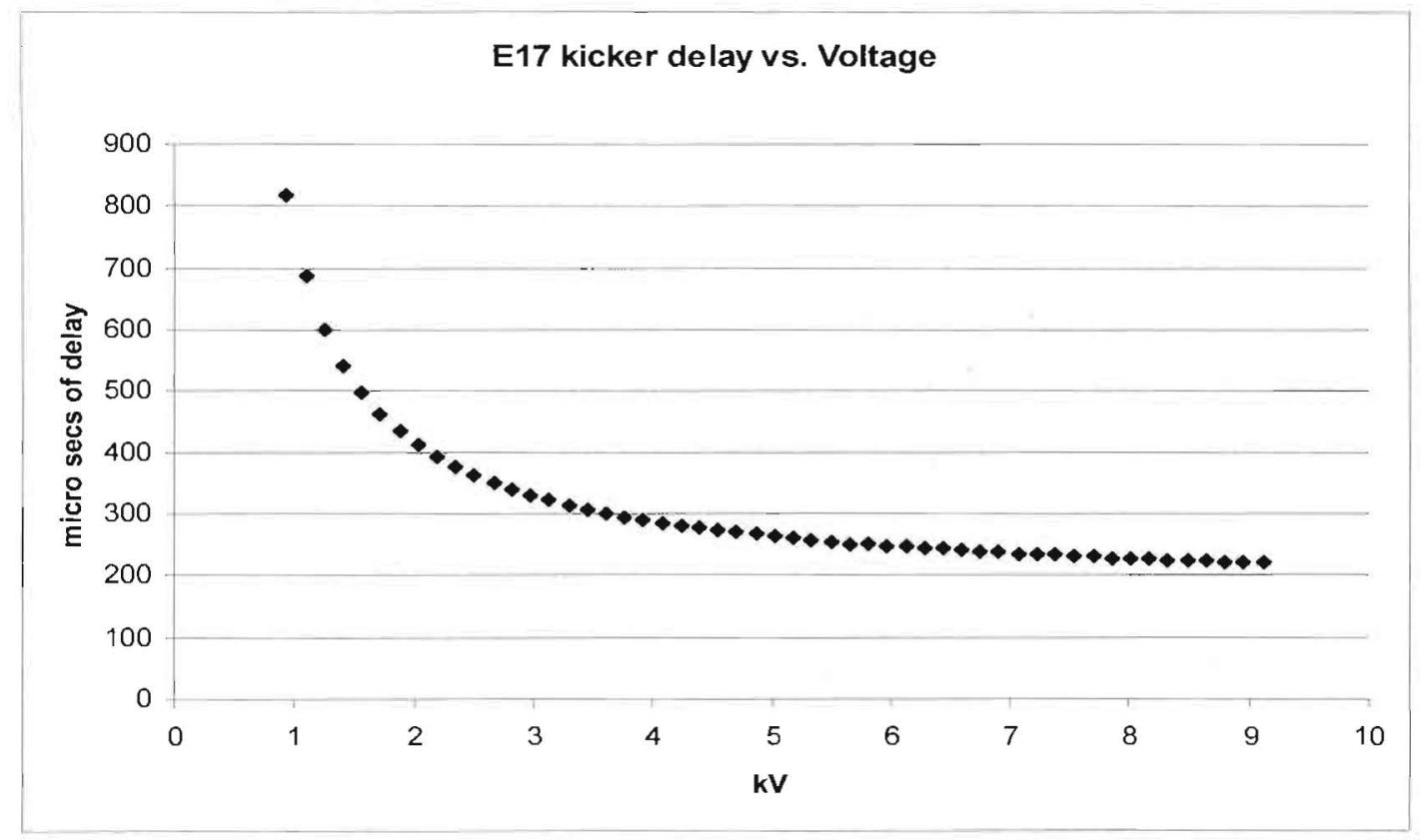

Figure 3: E17 kicker delay versus voltage.

\section{Results}

On several occasions we acquired data at $150 \mathrm{GeV}$ for a series of chromaticity settings with coalesced protons and later during the acceleration ramp. We typically used $1.5 \mathrm{~mm}$ (4.5p mm-mrad) kick. An example of the turn-by-turn data acquired after a typical kick is shown in Fig. 4. To extract the instantaneous phase for each turn for the selected two bunch slices a Hilbert transform was employed. This involves multiplying the 
signal $Z(n)$ by $2 \cos (2 \pi n Q)$ and $-2 \sin (2 \pi n Q)$ then filtering out the high frequency components $f<2 Q$ to obtain $y(n)$ and $x(n)$. From arctangent of $y(n)$ and $x(n)$ the phase as a function of turn number $\mathrm{n}$ can be found. We then can calculate $\Delta \Psi(\mathrm{n})$ using both slices. In Fig. 5 you can see an example of the evolution of $\Delta \Psi(\mathrm{n})$ over the a single synchrotron period.
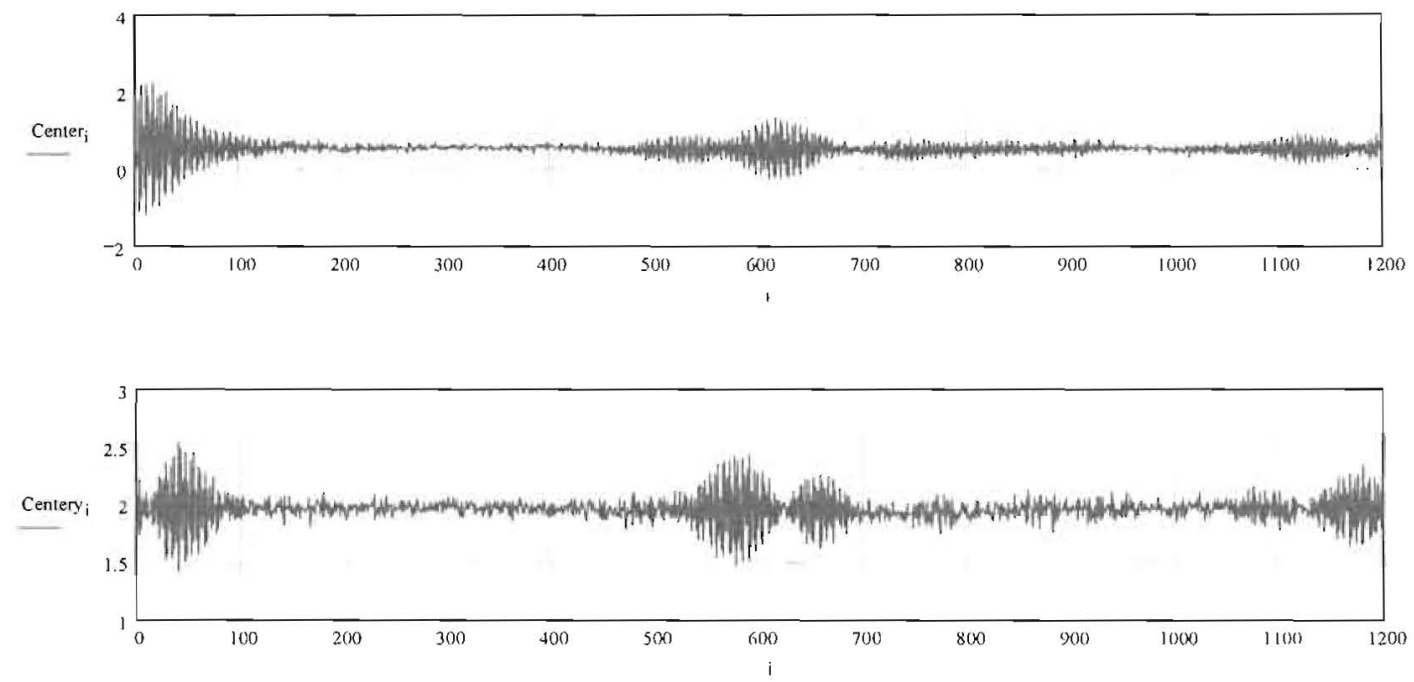

Figure 4: Horizontal (top) and vertical (bottom) turn-by-turn positions after $1.6 \mathrm{~mm}$ horizontal kick.
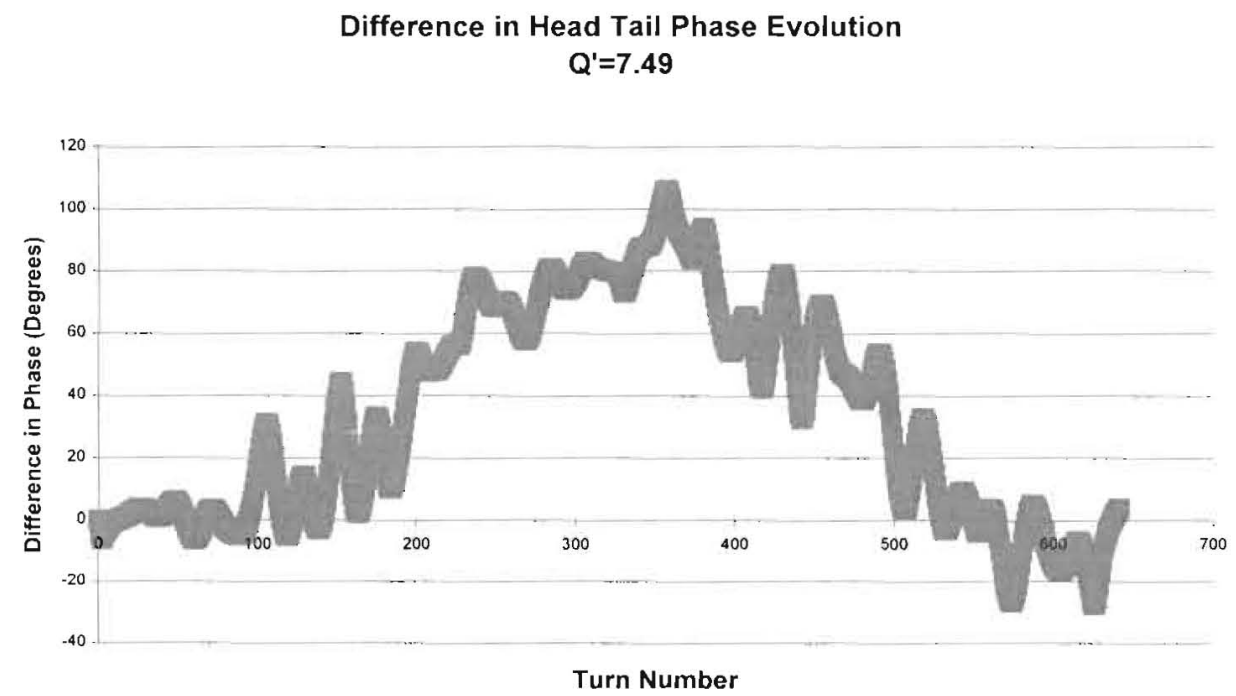

Figure 5: Evolution of $\Delta \Psi(n)$ over the first synchrotron period after horizontal kick. 


\section{Chromaticity Calculation \\ $Q^{\prime}=7.49$}

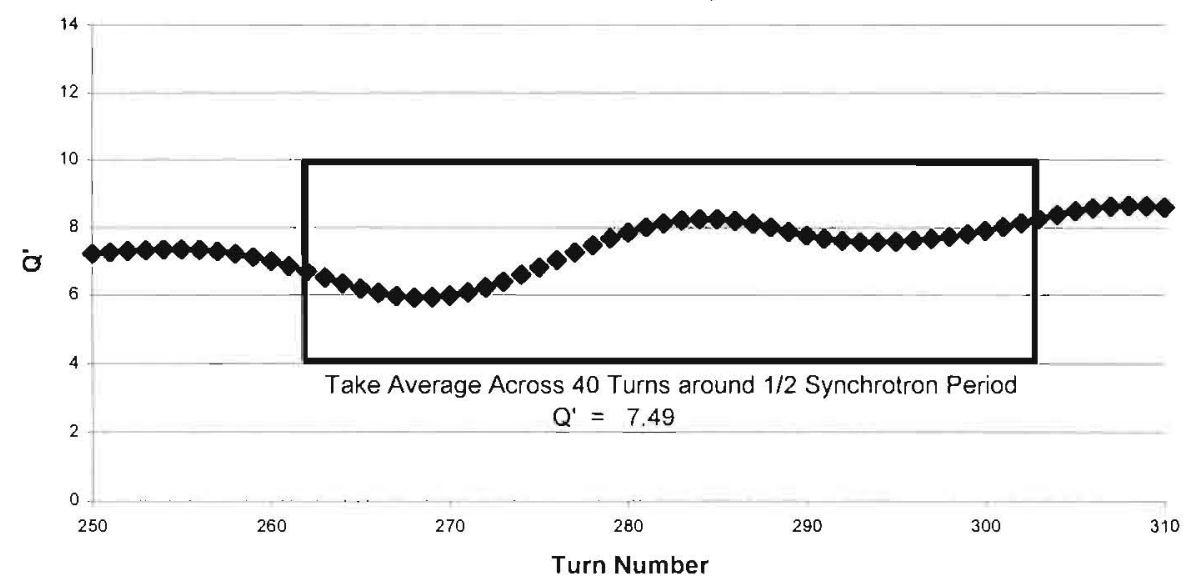

Figure 6: Chromaticity calculated around the $1 / 2$ synchrotron period.

Solving for the chromaticity for each turn we select 40 points during the middle of the synchrotron period to average over (see Fig. 6). Since the calculation goes as $1 /\left(\cos \left(2 \pi n Q_{s}\right)-1\right)$ the error should be a minimum at the $1 / 2$ synchrotron period point.

In Fig. 7 we compare the head-tail technique to the standard RF technique currently in use. The measurements using the RF technique were carried out using an uncoalesced proton bunch. In the horizontal plane the same chromaticity set points were then used for coalesced bunches. 
FERMILAB-FN-0753 June 2004
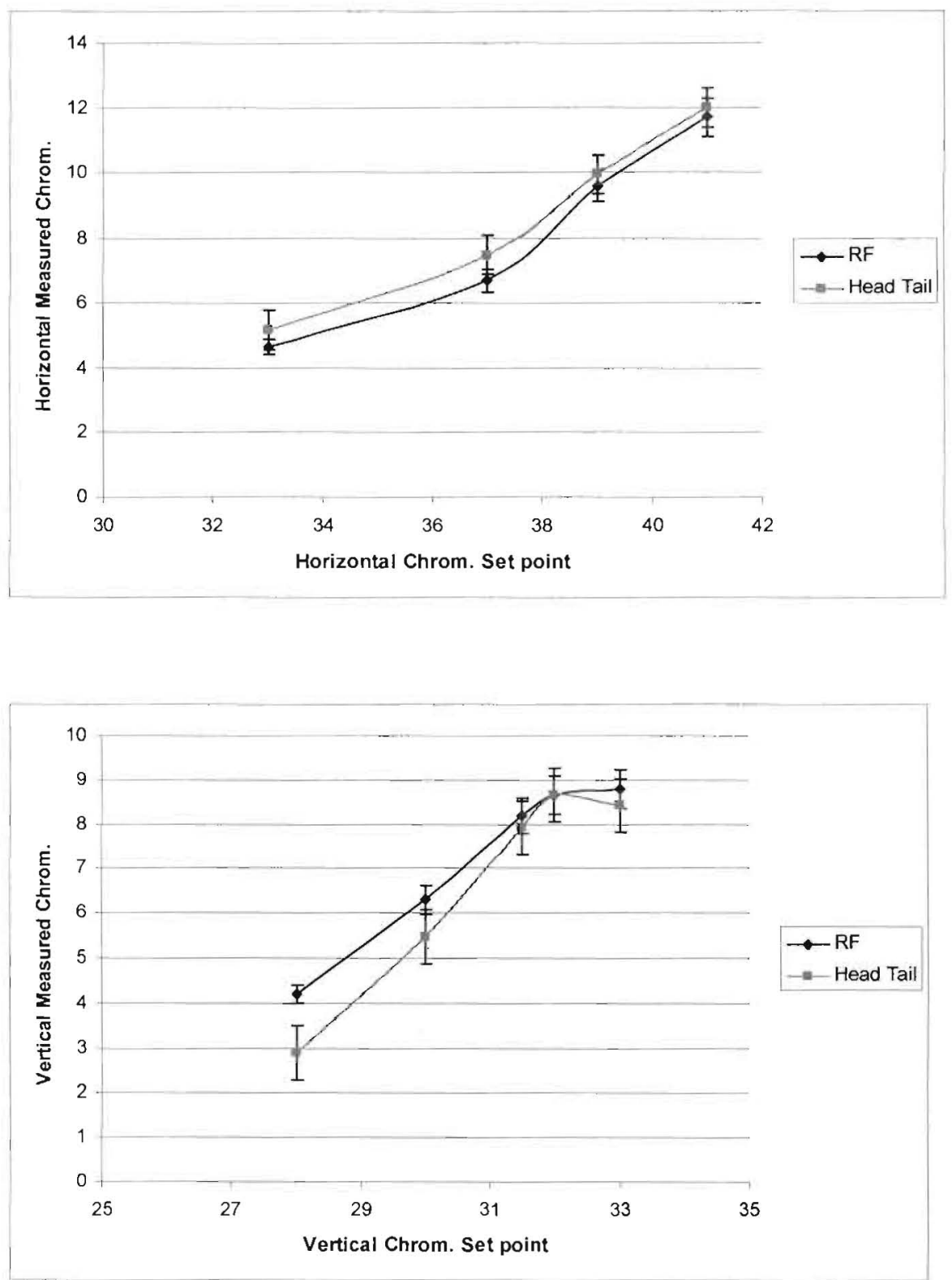

Figure 7: Horizontal (top) and Vertical (bottom) head-tail chromaticity measurement compared with RF chromaticity measurement at $150 \mathrm{GeV}$. 

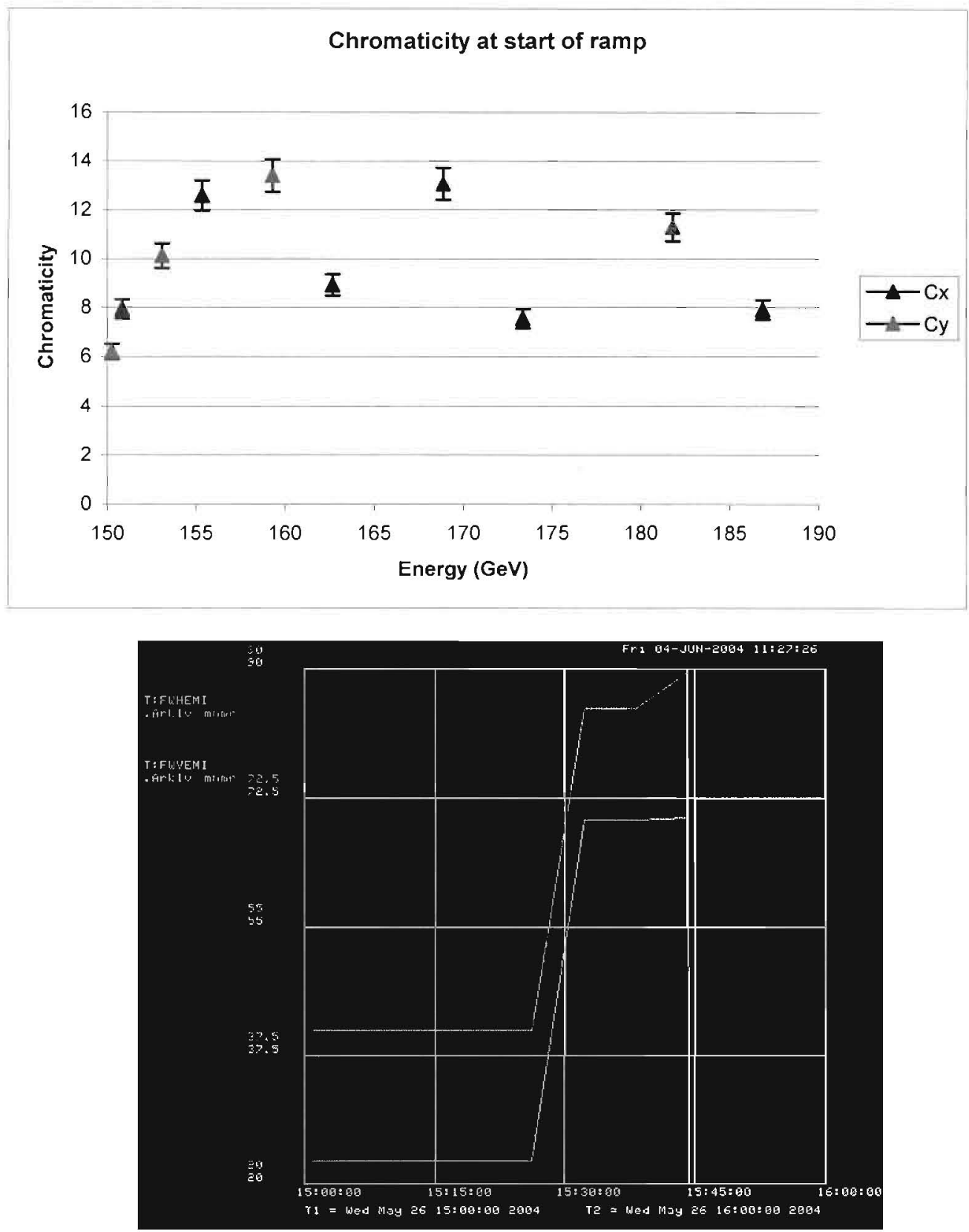

Figure 8: Horizontal (Cx) and Vertical (Cy) chromaticities during the first $12 \mathrm{sec}$ of the ramp (top graph) and the emittance growth of about $50 \pi \mathrm{mm}$-mrad in both planes as a result of the $104.5 \pi \mathrm{mm}$-mrad kick (bottom plot).

In Fig. 8 is an example of 10 points taken during the first 12 seconds of acceleration. These ten kicks caused at total of $50 \pi \mathrm{mm}$-mrad growth in the emittance of both planes. 


\section{C100 Console Application}

The $\mathrm{C} 100$ console head-tail chromaticity program opens with a GUI interface shown:

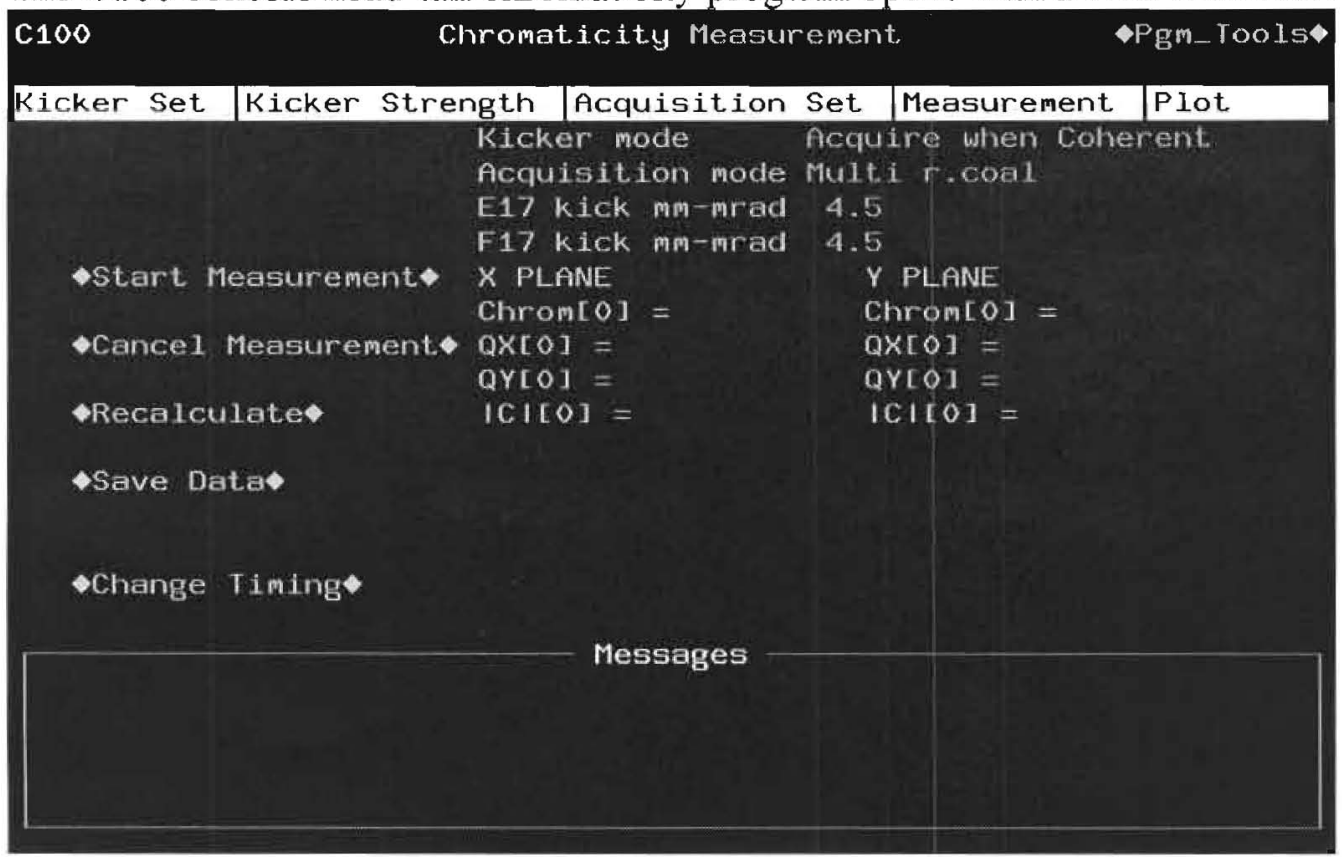

You can choose your kicker setting from the "kicker set" menu. Kick in horizontal, vertical or both planes (at different times).

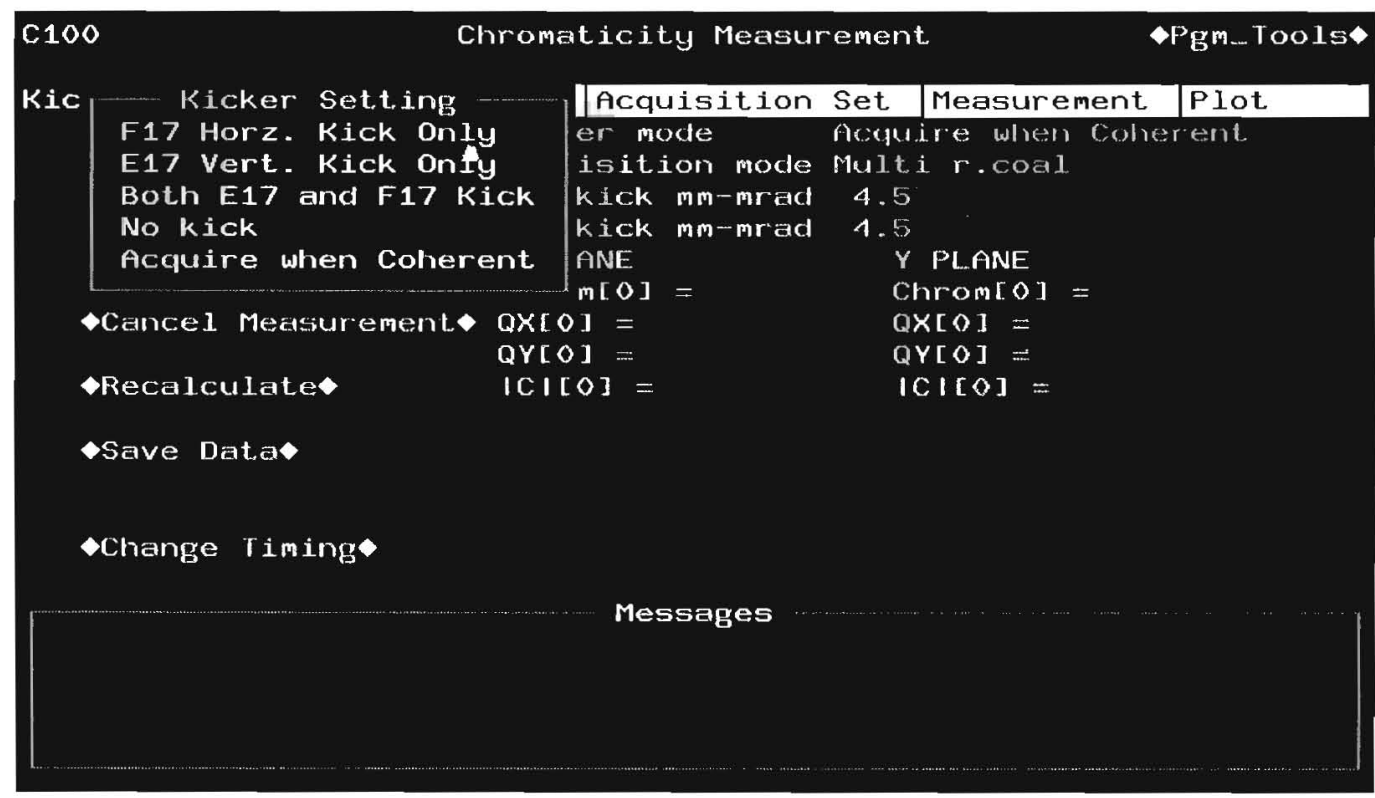




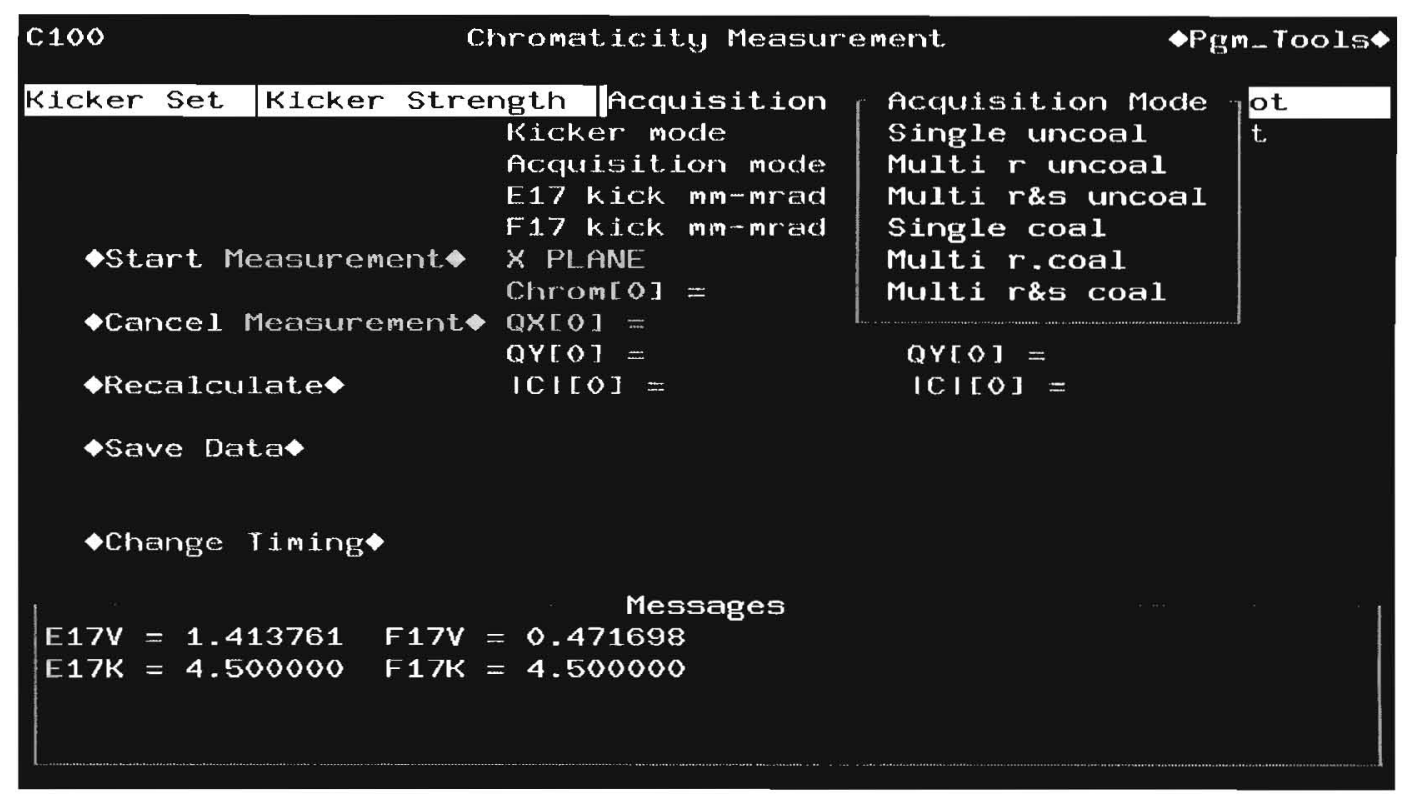

You can choose a multiple or single coalesced or uncoalesced acquisition. The multiple ramp and squeeze (r\&s) mode is not in use. If you need to take data during a squeeze use the ramp mode (r) with the squeeze event instead of the ramp event.

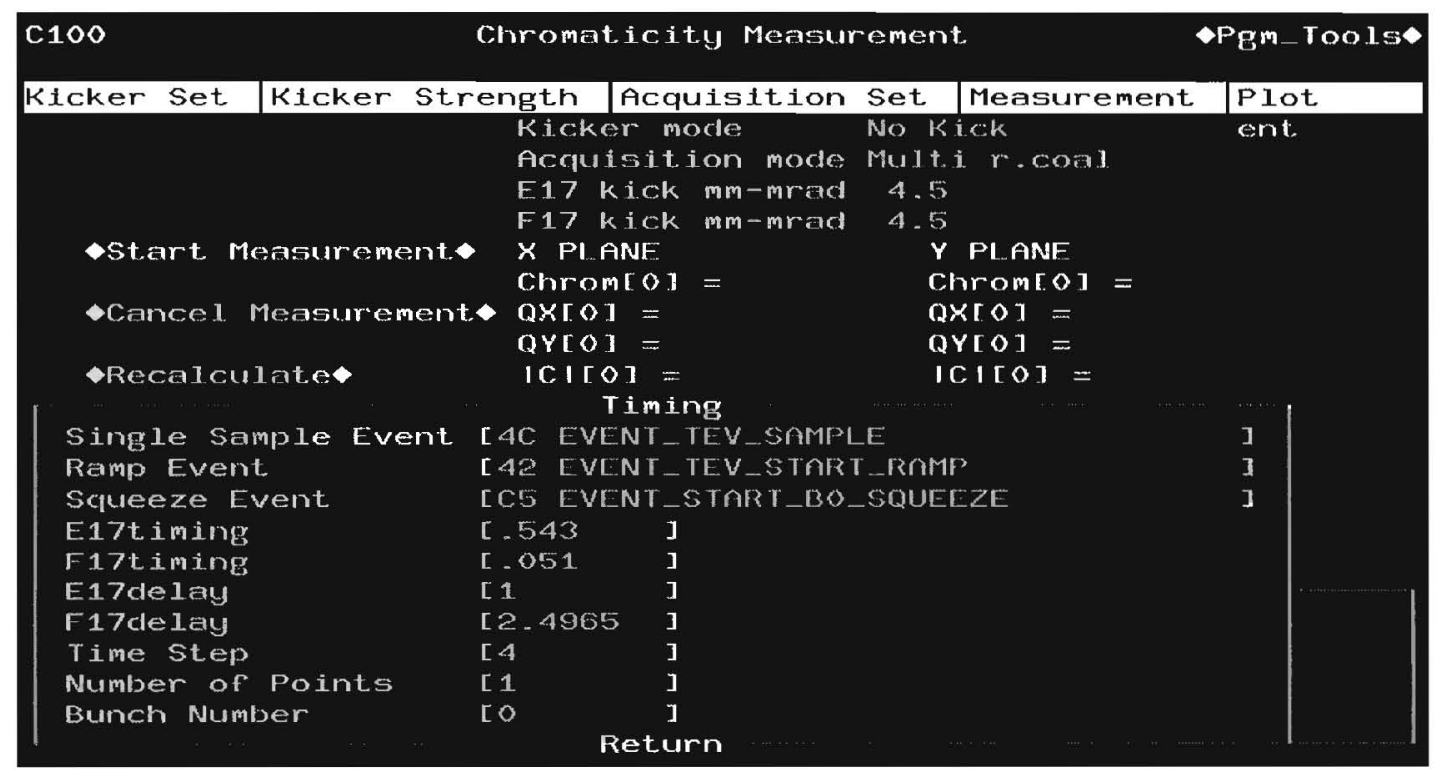

The "Change Timing" button will bring up the timing menu. If you are taking multiple measurements during the ramp leave the ramp event on 42. If you are taking multiple measurements during the squeeze change the ramp event to the squeeze event $\mathrm{C} 5$. 
The E17 and F17 delay amounts are measured in seconds from either the Single sample event or the ramp event. During a multiple ramp measurement the kickers first trigger on the ramp event then a $4 \mathrm{C}$ event triggers them there after at a time step given by "Time step" in seconds. Number of Points indicates the number of points you wish to measure in each plane. So if both planes are selected then number of points equal to five will yield 10 total measurements ( 5 horizontal 5 vertical). If only vertical or horizontal is selected then only five points will be sampled. The scope is capable of sampling only a total of 10 points in turn-by-turn mode and 20 in every-other-turn mode. After you have finished setting up the timing then to start a measurement press the "start measurement" button. When the measurement is over the first measured value will appear in the console screen. If multiple measurements are taken then in the message screen each measurement with its associated energy will be printed out. Note that measurements are only valid for the plane that was kicked. So even though both horizontal and vertical chromaticity values will be printed out for each measurement the only valid measurement is the one associated with the plane that was kicked.

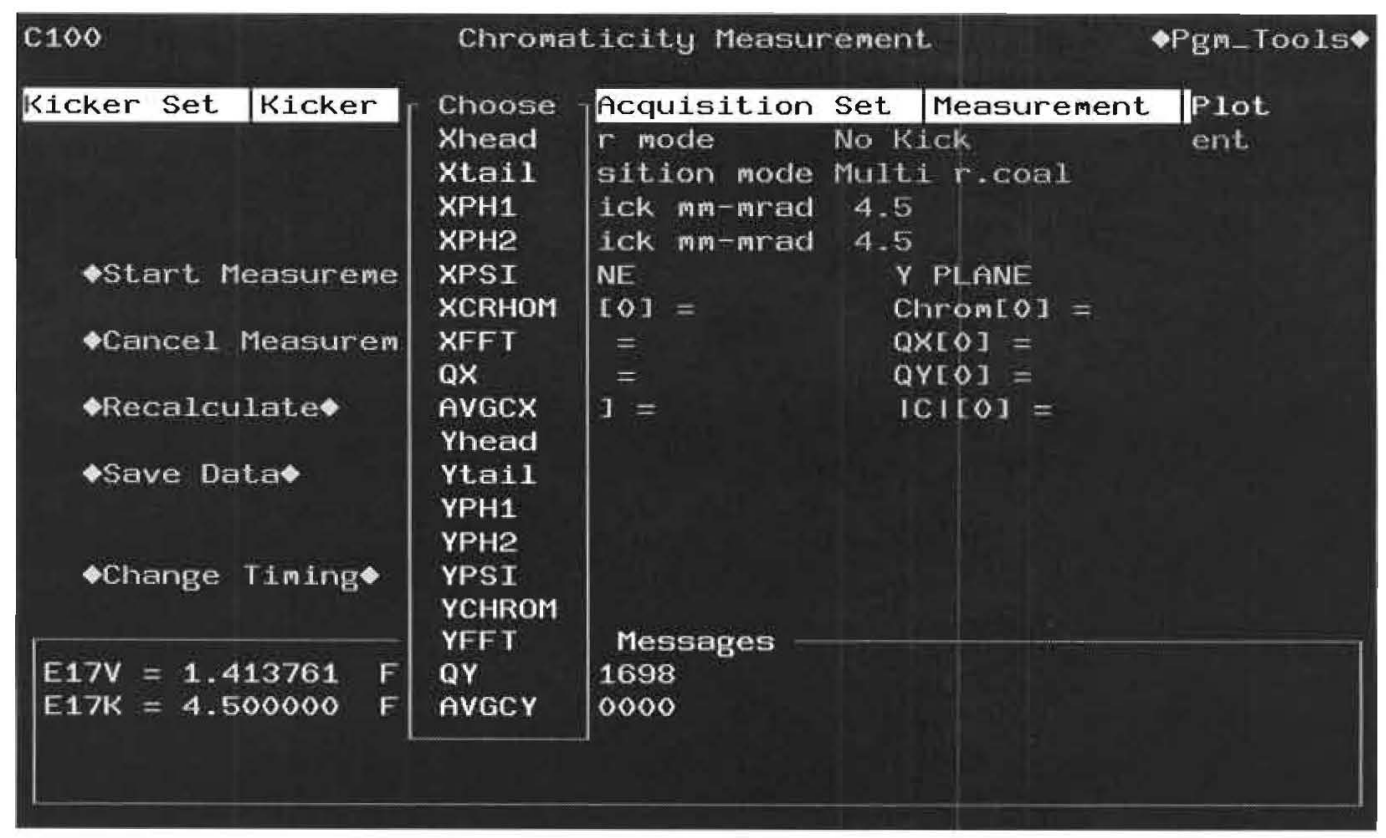

The plot menu allows you to choose to plot the head or tail horizontal or vertical turn-byturn positions (Xhead,Xtail,Yhead,Ytail) as well as the FFT (XFFT,YFFT) and head-tail 
phase difference (XPSI, YPSI). If you have taken multiple measurements then all the turn-by-turn data can be plotted at once by using the Measurement menu and setting the number of plots to the number, which you desire to see, plotted from the plot menu.

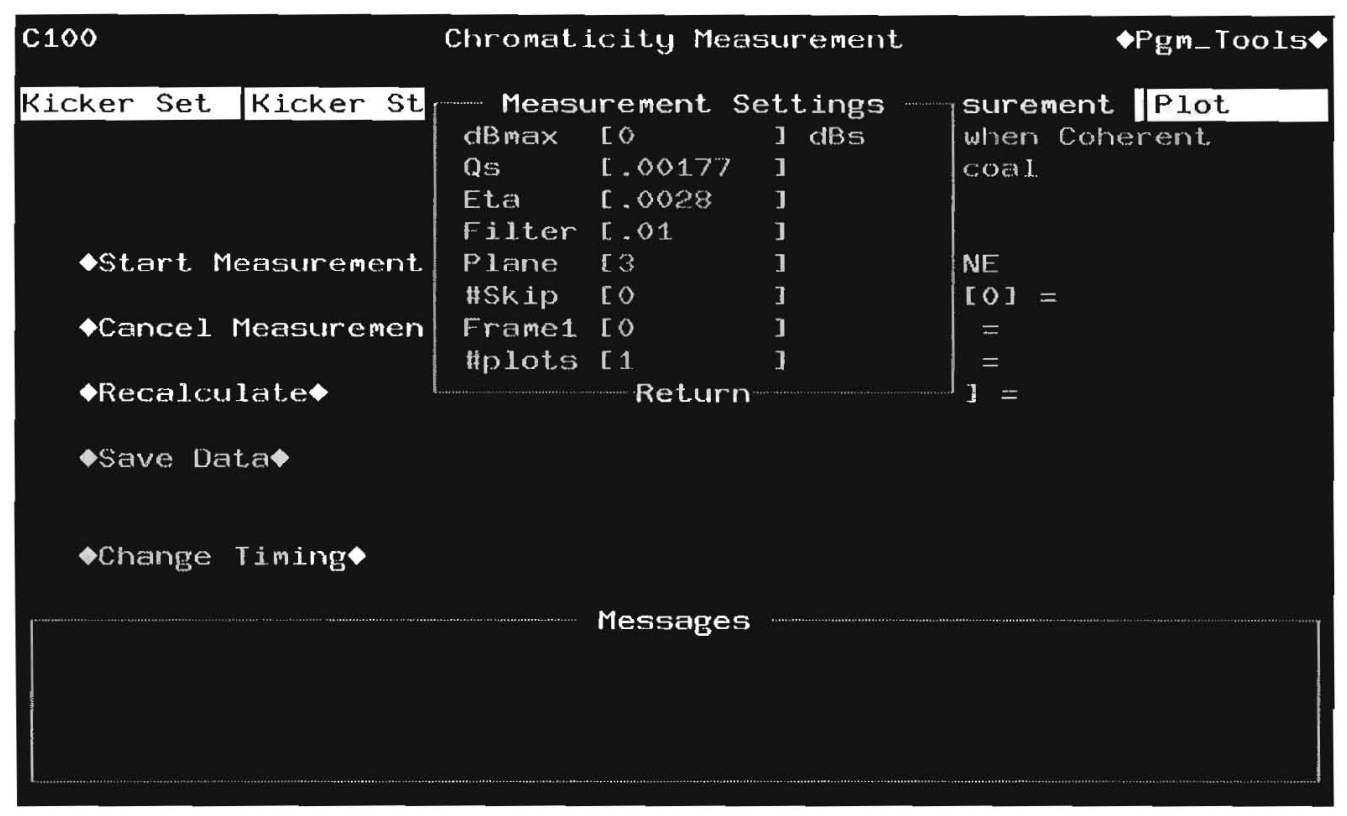

After taking a measurement the data can then be saved to the vax under cbs_files:[cns_write.studies.tbt_studies]Chr\%s.dat (with \%= date and time). After a measurement is complete pressing "Cancel Measurement" will remove all the events and turn off all the kickers.

\section{References}

[1] D. Cocq, O. R. Jones, H. Schmickler, The Measurement of Chromaticity Via a Head-Tail Phase Shift, 8th Beam Instrumentation Workshop (BIW'98), Stanford, CA, USA, May 1998. S. Fartoukh and R. Jones, Determination of Chromaticity by the Measurement of Head-Tail Phase Shifts: simulations, results from the SPS and a robustness study for the LHC, LHC Project Report 6022002. 